\title{
Appendix B
}

\section{Graphs, posets, and topological invariants}

The purpose of this appendix is to give a minimal required system of definitions and mathematical constructions needed to understand and to follow the discussion of the visualization of lattices by graphs and calculation of corresponding topological invariants introduced in Chapter 6, section 6.7.

We start by several intuitively evident but important definitions.

A graph $G=(V, E)$ consists of a finite set $V$ of vertices (nodes) and a finite set $E$ of edges. Every edge $e \in E$ consists of a pair of vertices, $u$ and $v$, called its endnodes. We will denote the edge $e$ by $u v$. Two vertices are said to be adjacent if they are joined by an edge. We will mainly consider here simple graphs, i.e. graphs in which every edge has distinct endnodes (no loops) and no two edges have the same two endnodes (no parallel or multiple edges). When every two nodes in $G$ are adjacent, the graph $G$ is said to be a complete graph. The complete graph on $n$ nodes is usually denoted by $K_{n}$.

Let $G=(V, E)$ be a graph. A graph $H=(W, F)$ is said to be a subgraph of $G$ if $W \subset V$ and $F \subset E$. Given an edge $e \in E$ in $G, G \backslash e:=(V, E \backslash e)$ is called the graph obtained from $G$ by deleting $e$.

Contracting an edge $e:=u v$ in $G$ means identifying the endpoints $u$ and $v$ of $e$ and deleting the parallel edges that may be created while identifying $u$ and $v . G / e$ denotes the graph obtained from $G$ by contracting the edge $e$. For an edge set $F \subseteq E, G / F$ denotes the graph obtained from $G$ by contracting all edges of $F$ (in any order).

Two graphs $G=(V, E)$ and $G^{\prime}=\left(V^{\prime}, E^{\prime}\right)$ are said to be isomorphic, $G \simeq$ $G^{\prime}$, if there exists a bijection $f: V \rightarrow V^{\prime}$ such that $u v \in E \Leftrightarrow f(u) f(v) \in E^{\prime}$.

A graph is said to be connected if, for every two nodes $u, v \in G$, there exists a path in $G$ joining $u$ and $v$. The rank of the graph is the number of nodes minus the number of connected components. 


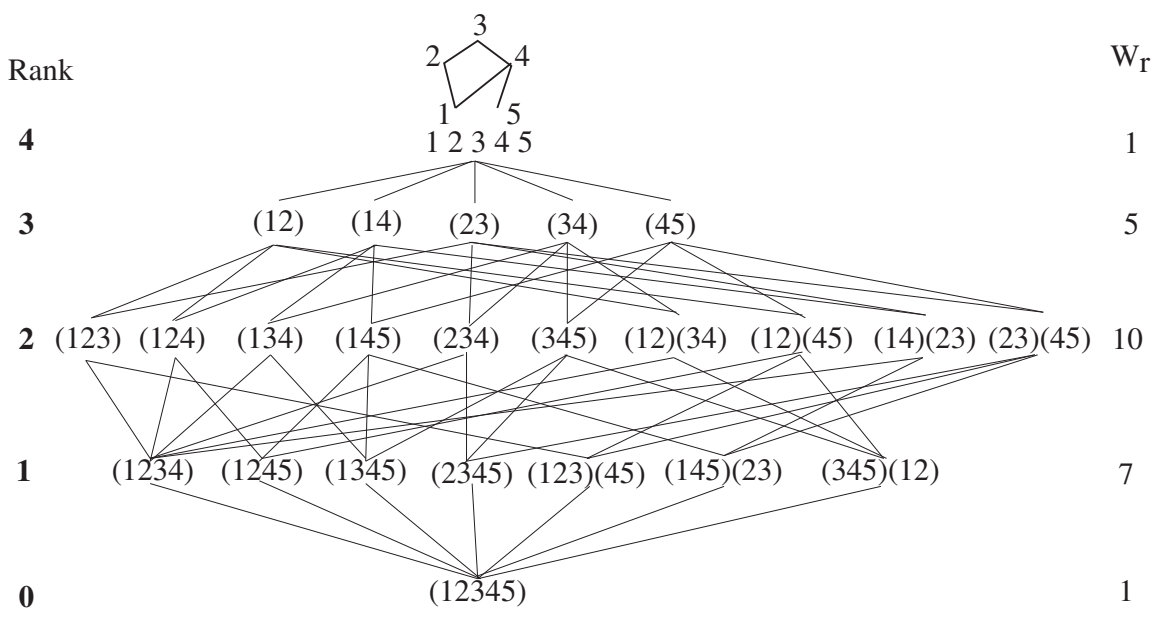

FIG. B.1 - Ranked partially ordered set of contractions for a graph representing the 14.28-0 4-d lattice. Only fused vertices are shown for contracted subgraphs. Lines symbolize the partial order imposed by contractions.

For a given simple connected graph we can construct all graphs which can be obtained from the initial graph by one or several contractions of edges. The set of so obtained graphs form a partially ordered set (see definition of partially ordered set or poset in appendix A). An example of such a partially ordered set (Poset) is shown in Figure B.1. In Figure B.1 only the initial graph is shown. Graphs obtained by contractions of the initial graph are represented just by labels of nodes which were fused during the contraction. The rank is well defined for all contracted graphs. Contracting one edge decreases rank by one. Thus we obtain the so called ranked partially ordered set of contractions, $P$. There are some number of topological invariants which can be introduced for the ranked partially ordered set $P$.

The simplest invariant is the number of elements of rank $k$ of the poset $P$. This invariant is named a simply indexed Whitney number of second kind, $W_{k}$. More complicated invariants are the doubly indexed Whitney numbers, $W_{i j}$ of the second kind. In order to introduce them we need to study subsets of $P$ with rank $r=i$ and with rank $r=j$. Whitney number $W_{i j}$ of the second kind gives the number of pairs $\left\{\left(x^{i}, x^{j}\right): x^{i} \leq x^{j}\right\}$ of elements of $P$ which satisfy the order relation. If $i$ and $j$ are neighboring integers, the corresponding Whitney number is just equal to the number of contraction lines between neighboring rows of a poset. It is clear that simply indexed Whitney numbers are a special case of doubly indexed ones, namely $W_{0, j}=W_{j}$. 
From Figure B.1 we immediately find the doubly indexed Whitney numbers which can be represented in the form of a triangular table

\begin{tabular}{cccccc}
\hline Graph & $W_{00}$ & $W_{i 1}$ & $W_{i 2}$ & $W_{i 3}$ & $W_{i 4}$ \\
\hline \multirow{3}{*}{$\mathbf{1 4 . 2 8 - 0}$} & 1 & 7 & 10 & 5 & 1 \\
& & 7 & 24 & 22 & 7 \\
& & & 10 & 20 & 10 \\
& & & & 5 & 5 \\
\hline
\end{tabular}

which shows some equivalence between $W_{i j}$ values which remains valid for a wide class of graphs.

The formal definition of the doubly indexed Whitney numbers of the second kind can be written as follows

$$
W_{i j}(P)=\left|\left\{\left(x^{i}, x^{j}\right): x^{i} \leq x^{j}\right\}\right|,
$$

where $|S|$ means the cardinality of the set $S$, i.e. the number of elements in the set.

The construction of the Whitney numbers of the first kind is based on the preliminary introduction of the Möbius function for a ranked partially ordered set. To be maximally concrete we restrict ourselves always to posets of contractions for a simple connected graph, which is one of the subgraphs of a complete graph $K_{n}$. We start by calculating values of the Möbius $\mu$-function for all elements of the poset $P$. To find these values we use $\mu(g, g)=1$ for the initial graph $g$. Next, for $b \neq g$ we calculate $\mu(b, 0)$ as a sum

$$
\mu(b, 0)=-\sum_{g \geq c>b} \mu(c, g),
$$

over all $c$ which are partially ordered with respect to $b$ and are strictly greater than $b$. The result of this calculation is illustrated in Figure B.2, upper right subfigure; it gives a system of simply indexed Whitney numbers of the first kind, $w_{i}=w_{0 i}$. Generalization to doubly indexed Whitney numbers of the first kind is similar to what we have done for Whitney numbers of the second kind.

To calculate doubly indexed Whitney numbers of the first kind $w_{k i}$ we need to analyze only the sub-poset of the initial poset taking into account the elements with the rank not exceeding $r-k$ where $r$ is the rank of the initial graph, and calculate $\mu$-values for this sub-poset. The lower left sub-figure of B.2 visualizes the "neglected" part of the initial poset by using dashed lines. For this sub-poset we calculate $\mu(b)$ values with respect to the rank 2 level. This explains why for all $b=(i j)$ elements now $\mu(b)=1$ and $w_{11}=4$. Going to the lower rank $r=1$ we see that the value of $\mu(123)$, for example, should be calculated with respect to the level with the rank equal 2 and we have only three contributions from $(12),(13),(23)$ elements which all equal -1 . 

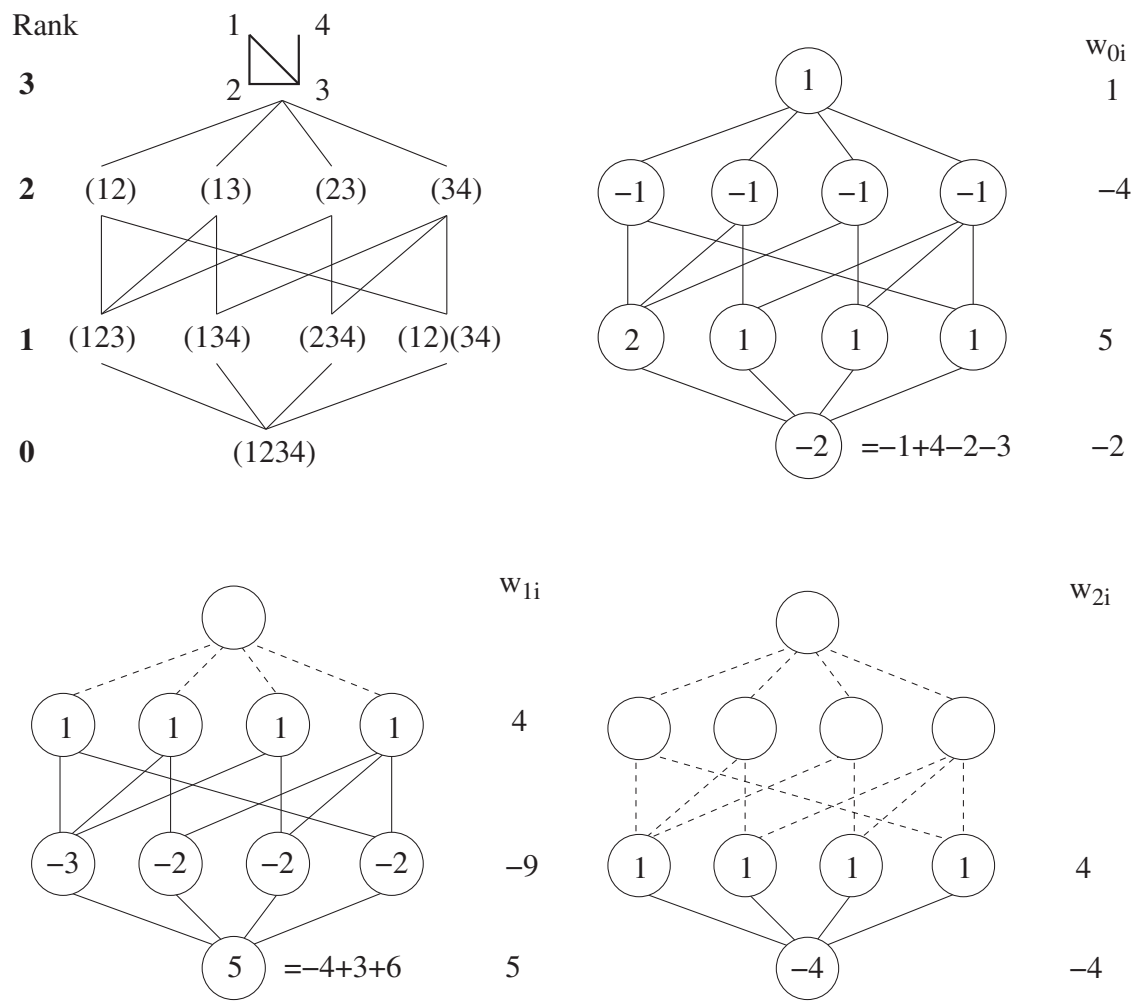

$\mathrm{w}_{1 \mathrm{i}}$

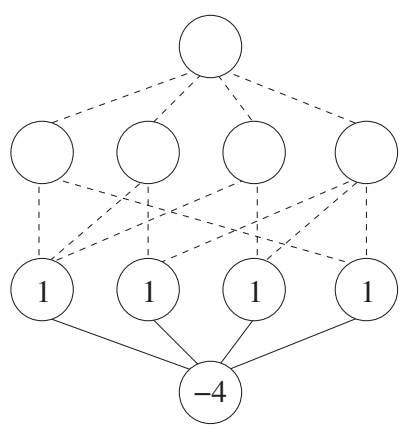

$\mathrm{w}_{2 \mathrm{i}}$

4

5

4

FIG. B.2 - A ranked partially ordered set of contractions for a graph representing the 8.12 3-d lattice. The upper left subfigure shows initial graph and for each contraction step indicates fused vertices for contracted graphs. Lines symbolize the partial order imposed by contractions. The upper right subfigure reproduces the same poset and for each element $a$ shows the value of the Möbius function $\mu(a, 0)$. On the right of the poset the values $w_{0 i}=w_{i}$ of the Whitney numbers of the first kind are given which are the sum of $\mu$-values for all elements of the same rank. In a similar way the lower left and right subfigures illustrate calculation of the Whitney numbers of the first kind for $w_{1 i}$ and for $w_{2 i}$.

Completing calculations for all elements of rank 1 we get $w_{12}=-9$ and in a similar way we get $w_{13}=5$.

Finally, to calculate Whitney number $w_{2 i}$ we need to study only the subposet of the initial poset taking into account the elements with the rank not exceeding 1 and calculate $\mu$-values for this sub-poset. Lower right sub-figure of B.2 visualizes the "neglected" part and indicates corresponding $\mu(b)$ values and $w_{2 i}$. 
TAB. B.1 - Doubly indexed Whitney numbers of the first and second kind for several graphs corresponding to some of four-dimensional zonotopes.

\begin{tabular}{|c|c|c|c|c|c|c|c|c|c|}
\hline Graph & $w_{00}$ & $w_{i 1}$ & $w_{i 2}$ & $w_{i 3}$ & $w_{i 4}$ & $\sum_{j} w_{i j}^{+}$ & $W_{01}$ & $W_{02}$ & $W_{03}$ \\
\hline \multirow{5}{*}{ 8.16-0 } & \multirow[t]{5}{*}{1} & -4 & 6 & -4 & 1 & $16=N_{0}$ & 4 & 6 & 4 \\
\hline & & \multirow[t]{4}{*}{4} & -12 & 12 & -4 & $32=N_{1}$ & \multirow[t]{4}{*}{4} & 12 & 12 \\
\hline & & & 6 & -12 & 6 & $24=N_{2}$ & & 6 & 12 \\
\hline & & & & 4 & -4 & $8=N_{3}$ & & & 4 \\
\hline & & & & & 1 & & & & \\
\hline \multirow{5}{*}{ 20.30-0 } & \multirow[t]{5}{*}{1} & -5 & 10 & -10 & 4 & $30=N_{0}$ & 10 & 10 & 5 \\
\hline & & 5 & -20 & 30 & -15 & $70=N_{1}$ & 10 & 30 & 30 \\
\hline & & & 10 & -30 & 20 & $60=N_{2}$ & & 10 & 20 \\
\hline & & & & 10 & -10 & $20=N_{3}$ & & & 5 \\
\hline & & & & & 1 & & & & \\
\hline \multirow{5}{*}{ 12.36-12 } & \multirow[t]{5}{*}{1} & -6 & 13 & -12 & 4 & $36=N_{0}$ & 6 & 11 & 6 \\
\hline & & 6 & -24 & 30 & -12 & $72=N_{1}$ & 6 & 24 & 24 \\
\hline & & & 11 & -24 & 13 & $48=N_{2}$ & & 11 & 24 \\
\hline & & & & 6 & -6 & $12=N_{3}$ & & & 6 \\
\hline & & & & & 1 & & & & \\
\hline \multirow{5}{*}{ 22.46-0 } & \multirow[t]{5}{*}{1} & -6 & 15 & -17 & 7 & $46=N_{0}$ & 11 & 15 & 6 \\
\hline & & 6 & -30 & 48 & -24 & $108=N_{1}$ & 11 & 42 & 36 \\
\hline & & & 15 & -42 & 27 & $84=N_{2}$ & & 15 & 30 \\
\hline & & & & 11 & -11 & $22=N_{3}$ & & & 6 \\
\hline & & & & & 1 & & & & \\
\hline \multirow{5}{*}{ 24.60-12 } & \multirow[t]{5}{*}{1} & -7 & 19 & -23 & 10 & $60=N_{0}$ & 12 & 17 & 7 \\
\hline & & 7 & -36 & 60 & -31 & $134=N_{1}$ & 12 & 49 & 44 \\
\hline & & & 17 & -49 & 32 & $98=N_{2}$ & & 17 & 36 \\
\hline & & & & 12 & -12 & $24=N_{3}$ & & & 7 \\
\hline & & & & & 1 & & & & \\
\hline & \multirow[t]{5}{*}{1} & -10 & 35 & -50 & 24 & $120=N_{0}$ & 15 & 25 & 10 \\
\hline $\mathbb{B}$ & & 10 & -60 & 110 & -60 & $240=N_{1}$ & 15 & 75 & 7 \\
\hline \multirow{3}{*}{ 30.120-60 } & & & 25 & -75 & 50 & $150=N_{2}$ & & 25 & 60 \\
\hline & & & & 15 & -15 & $30=N_{3}$ & & & 10 \\
\hline & & & & & 1 & & & & \\
\hline
\end{tabular}


Table B.1 gives Whitney numbers of the first and of the second kind for several four-dimensional zonotopes. Face numbers for corresponding zonotopes are given along with Whitney numbers of the first kind because of the simple relation between face numbers and Whitney numbers of the first kind. Namely, for subgraphs of $K_{r+1}$ representing zonotopes we have [56]

$$
\sum_{j=k}^{r} w_{k j}^{+}=N_{k}
$$

where $w_{k j}^{+}=\left|w_{k j}\right|$ and $N_{k}$ are the number of $k$-faces of the zonotope associated with the graph.

Only $W_{i 1}, W_{i 2} W_{i 3}$ are shown in table B.1 for the Whitney numbers of the second kind. The rest of the table can be easily reconstructed taking into account the symmetry of the table, namely $W_{i i}=W_{i 4}$ and $W_{44}=W_{00}=1$.

We note also that the singly indexed Whitney numbers of the first kind are the coefficients of the chromatic polynomial. The chromatic polynomial

$$
P_{G}(t)=\sum_{k=0}^{r} w_{0 k} t^{r+1-k}
$$

shows how many different coloring of graph nodes are possible with $t$ colors with the restriction on adjacent nodes to be of different color.

For more details on relevant material see [1, 20, 9]. 